\title{
Inactivity on the kidney transplant wait-list is associated with inferior pre- and post- transplant outcomes
}

\author{
Norman SP, Kommareddi M, Luan FL. Inactivity on the kidney \\ transplant wait-list is associated with inferior pre- and post- \\ transplant outcomes.
}

\begin{abstract}
Background: The majority of kidney transplant (KT) candidates spend some time on the transplant wait-list (WL) prior to kidney transplantation. We examined the impact of WL inactivity on clinical outcomes.

Methods: All adult KT candidates first actively wait-listed between January 1, 1996, and December 31, 2005, in the United States were grouped by frequency of inactivity on the WL. Transplantation rate, preand post-transplant patient survival and death-censored kidney graft survival were compared.

Results: Of 159774 candidates who were placed on the WL, 48598 (30.4\%) experienced one or more periods of inactivity. Candidates with inactivity once or more on the WL had $42 \%$ and $27 \%$ less likelihood of KT, respectively (HR $0.58,95 \%$ CI $0.57,0.59$ and HR $0.73,95 \%$ CI 0.71 , $0.75)$. WL inactivity once or more was associated with a higher likelihood of death (HR 1.94, 95\% CI 1.88, 2.00 and HR 2.13, 95\% CI 2.02, 2.24).

Among KT recipients, inactivity more than once on the WL was associated with a higher risk of death (HR 1.14, 95\% CI 1.05, 1.23). Conclusions: Periods of inactivity on the WL predict increased mortality pre- and post-transplantation. A better understanding of the reasons for WL inactivity is essential to improve WL management and posttransplant outcomes.
\end{abstract}

\section{Silas Prescod Norman, Mallika Kommareddi and Fu Lung Luan}

Division of Nephrology, Department of Internal Medicine, University of Michigan, Ann Arbor, MI, USA

Key words: inactivity - kidney - survival transplant - wait-list

Corresponding author: Silas Prescod Norman, MD, 3914 Taubman Center, Box 0364, 1500 East Medical Center Drive, Ann Arbor, MI 48109-0364, USA.

Tel.: 734763 9041; fax: 734232 4160; e-mail: spnorman@med.umich.edu

Conflict of interest: The authors have no conflict of interest to disclose.

Accepted for publication 21 May 2013
Kidney transplantation is the best treatment modality for qualified patients with end-stage renal disease (ESRD) and is associated with improved patient survival and quality of life compared with dialysis $(1,2)$. There are several steps involved to receive a kidney transplant (KT) including patient referral, evaluation and wait-listing for transplantation. Previous studies have examined the factors that affect likelihood of transplant evaluation referral, completion of evaluation, and wait-listing $(3,4)$. Demographic differences in race and gender, and socioeconomic status have been consistently highlighted as factors among others that lead to differential access to be placed on the wait-list (WL; 4-7). Less attention has been paid to the impact of events that occur during wait-listing period on patient outcomes, despite the long period of time that qualified candidates often spend on the WL.

Once on the WL, the candidates can remain "active" (status 1 in $\mathrm{UNET}^{\mathrm{sm}}$ ) with the possibility of receiving a KT when an organ becomes available, or be temporarily "inactive" (status 7) which makes them ineligible to receive a KT. A candidate may change from active to inactive status several times prior to successful transplantation. Candidates are ultimately removed from the WL for one of three reasons: transplant, death, or delisting. While practices vary among the different transplant programs, the placement of a previously active candidate into an inactive status typically reflects a medical or non-medical acute event that makes the candidate temporarily inappropriate for transplantation with the expectation that the candidate will be appropriate for transplant once the acute event resolves. The prevalence of "inactive" candidates in a given year has been increasing in recent years likely reflecting an increase in overall medical co-morbidities (8). How inactive status on the WL impacts clinical outcomes before and after kidney transplantation remains unclear. We hypothesized that the transition from the active to inactive status 
on the WL may be a surrogate marker for overall medical co-morbidity and/or complexity. We investigated the effect of WL inactivity on the clinical outcomes pre- and post-transplantation in a national cohort of wait-listed KT candidates.

\section{Materials and methods}

Using a US national transplant database provided by the Organ Procurement and Transplantation Network (OPTN)/Scientific Registry of Transplant Recipients (SRTR), we identified all adult (age $\geq 18$ ) KT candidates who were first time actively wait-listed between January 1, 1996, and December 31, 2005. Study follow-up continued until January 31, 2010. The SRTR data system includes data on all donors, wait-listed candidates, and transplant recipients in the United States, submitted by the members of the OPTN, and has been described elsewhere (9). The Health Resources and Services Administration (HRSA), US Department of Health and Human Services, provides oversight to the activities of the OPTN/SRTR contractors. For each candidate, we determined whether during the wait-listing period he or she ever had a change from active (status 1) to inactive status (status 7), and if so, how many times. We separated the study population into three groups: (i) active - never inactivated; (ii) inactivated once; and (iii) inactivated more than once on the WL. We excluded candidates who never had been active on the WL, listed for and/or received multi-organ transplants, repeat KT, and candidates and patients with missing wait-listing and/or subsequent transplant outcome information. The primary outcomes of the study were the rate of kidney transplantation, mortality on the WL, and death-censored post-transplant kidney graft and recipient survival.

Baseline demographics of the study groups were compared using chi-square test and ANOVA, as appropriate. An unadjusted Poisson regression model was utilized to compare the incidence rate of receiving a KT, living and/or deceased, among the three groups on the WL taking into consideration the total time spent on the WL. Kaplan-Meier methods with log-rank test were used to estimate kidney graft and recipient survival following kidney transplantation. Two sets of Cox proportional hazard models were applied for clinical outcomes before and after kidney transplantation. For the primary outcomes of time to transplantation, censored for delisting and death, and time to the WL death, censored for delisting and transplantation, the time at risk began when candidates were actively wait-listed for the first-time with wait-listing inactivity as a two-tier time-dependent covariate. As some candidates started out as inactive on the WL and subsequently moved to active status, the time interval between these two was calculated and used as one of the covariates in the multivariate analyses as well. For kidney graft and recipient survival analyses, the time at risk began with the transplantation. The demographic and baseline variables at the time of active listing and transplantation were used for multivariate analyses, respectively. The interaction term was considered and tested wherever appropriate in multivariate analyses for pre- and post-transplant mortality risk. Final models contain those variables with $\mathrm{p}<0.10$ after backward selection.

Analyses were run in SAS 9.2 (SAS Institute Inc., Cary, NC, USA), with statistical significance set at a two-sided $\alpha=0.05$. The procedures of our study were in accordance with the Helsinki Declaration of 1975 .

\section{Results}

During the study period, a total of 163802 candidates were actively wait-listed for a first-time KT. After excluding multi-organ transplant candidates and/or recipients $(n=3686)$, candidates who had missing follow-up information $(\mathrm{n}=331)$, and candidates erroneously identified as having died and then subsequently relisted $(\mathrm{n}=11)$, there were 159774 candidates available for analysis. The mean age of wait-listed candidates was 49.7 \pm 13.0 yr. African Americans (AA) accounted for $29.6 \%$ (47 240) and males for 59.4\% (94 904) of the study population. Among all WL candidates, $37823(23.7 \%)$ experienced one and 10775 $(6.7 \%)$ candidates experienced more than one period of WL inactivity. Demographic characteristics of the study population are shown in Table 1. In general, the candidates who had been placed on inactive status at least once while on the WL tended to be older, heavier, and more likely AA. Inactivated candidates were less often college-educated and experienced longer dialysis times and were more likely to be sensitized. Furthermore, candidates who experienced inactivity displayed more medical co-morbidities such as diabetes mellitus (DM) and cardiovascular disease (CVD).

The distribution of study outcomes among candidates in the three groups at the end of study follow-up are shown in Fig. 1. In the univariate analysis, compared with candidates never inactivated on the $\mathrm{WL}$, the overall transplantation rate was significantly lower for candidates who were inactive either once or more than once (407.8 $\pm 3.3,101.9 \pm 2.0$ and $90.2 \pm 3.1$ transplants per 1000 patient-years, respectively, $\mathrm{p}<0.001$ for both 
Wait-list inactivity and clinical outcomes

Table 1. Demographic and baseline characteristics of wait-list candidates

\begin{tabular}{|c|c|c|c|c|}
\hline & $\begin{array}{l}\text { Never inactive } \\
N=111176\end{array}$ & $\begin{array}{l}\text { Inactive once } \\
N=37823\end{array}$ & $\begin{array}{l}\text { Inactive }>\text { once } \\
N=10775\end{array}$ & $\mathrm{p}$ \\
\hline Age, mean (SD) & $49.3(13.2)$ & $50.8(12.7)$ & $50.2(12.5)$ & $<0.001$ \\
\hline African American, n (\%) & 30525 (27.5) & $13138(34.7)$ & $3577(33.2)$ & $<0.001$ \\
\hline Male, $n(\%)$ & $66340(59.7)$ & $22298(59.0)$ & $6266(58.2)$ & 0.001 \\
\hline $\mathrm{BMI}^{*}$, mean (SD) & $27.3(5.4)$ & $28.6(6.7)$ & $28.6(6.4)$ & $<0.001$ \\
\hline Education, n (\%) & & & & $<0.001$ \\
\hline Less than high school & 7607 (6.8) & $2943(7.8)$ & $757(7.0)$ & \\
\hline High school & $55595(50.0)$ & $20356(53.8)$ & $5605(52.0)$ & \\
\hline College or higher & $47974(43.2)$ & $14524(38.4)$ & $4413(41.0)$ & \\
\hline Private insurance, $\mathrm{n}(\%)$ & $52211(47.0)$ & $15230(40.3)$ & $4717(43.8)$ & $<0.001$ \\
\hline On dialysis, $\mathrm{n}(\%)$ & $46840(42.1)$ & $21488(56.8)$ & $6289(58.4)$ & $<0.001$ \\
\hline Dialysis duration, yr (SD) & $0.7(1.7)$ & $1.1(2.1)$ & $1.0(1.8)$ & $<0.001$ \\
\hline PRA, n (\%) & & & & $<0.001$ \\
\hline$<10$ & 89508 (80.5) & $31314(82.8)$ & $8895(82.6)$ & \\
\hline $10-80$ & $17099(15.4)$ & $4196(11.1)$ & $1314(12.2)$ & \\
\hline$>80$ & $4569(4.1)$ & $2313(6.1)$ & $566(5.3)$ & \\
\hline Diabetes mellitus, n (\%) & 39250 (35.3) & $15696(41.5)$ & $4281(39.7)$ & $<0.001$ \\
\hline Cardiovascular disease, n (\%) & 16466 (14.8) & $6676(17.7)$ & $1888(17.5)$ & $<0.001$ \\
\hline Blood transfusion & $45411(40.8)$ & $16416(43.4)$ & $4834(44.9)$ & $<0.001$ \\
\hline
\end{tabular}

*BMl $=$ body mass index, $\mathrm{kg} / \mathrm{m}^{2}$.

Fig. 1. Clinical outcome distribution among study group candidates.

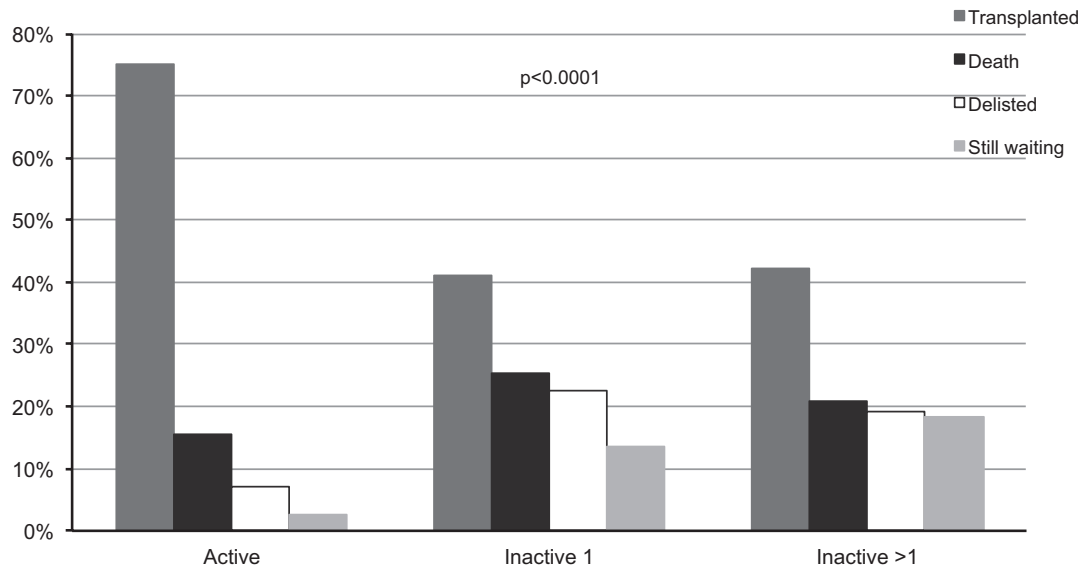

comparisons; Fig. 2). Inactivity negatively impacted transplant rates of both living and deceased donor KT. The effect of inactivity persisted even when only active time was considered to calculate transplant rates (data not shown). Multivariate Cox regression analyses with WL inactivity as a time-dependent variable showed that inactivity once or more than once while on the WL was associated with $42 \%$ and $27 \%$ lower likelihood of receiving a KT (HR $0.58,95 \%$ CI 0.57 , $0.59, \mathrm{p}<0.001$, and HR $0.73,95 \%$ CI $0.71,0.75$, $\mathrm{p}<0.001$, respectively). Other variables independently associated with the low likelihood of kidney transplantation included AA race (HR 0.50, 95\% CI $0.49,0.51, \mathrm{p}<0.001)$, medical co-morbidities such as DM (HR 0.83, 95\% CI 0.82, 0.84, $\mathrm{p}<0.001)$ or CVD (HR 0.92, 95\% CI 0.91, 0.94, $\mathrm{p}<0.001)$, lower education levels $(\mathrm{p}<0.001)$, and longer dialysis time $(\mathrm{p}<0.001)$ (Table 2$)$. In contrast, PRA level $>80$ (HR 1.20, 95\% CI 1.15, 1.26, $\mathrm{p}<0.001)$ and having a private insurance (HR $1.20,95 \%$ CI $1.19,1.22, \mathrm{p}<0.001)$ were associated with higher likelihood of receiving kidney transplantation.

We further analyzed mortality risk for all candidates on the WL according to their history of inactive status following the initial active listing, again using WL inactivity as a two-tier time-dependent variable. Compared with the candidates who were never placed in inactive status, the risk of WL death was $94 \%$ higher in candidates inactivated once (HR 1.94, 95\% CI 1.88, 2.00, p < 0.001) and $113 \%$ higher in candidates inactivated more than once (HR 2.13, 95\% CI 2.02, 2.24, p < 0.001). The high mortality risk was primarily contributed by death during inactive periods in the later follow-up 


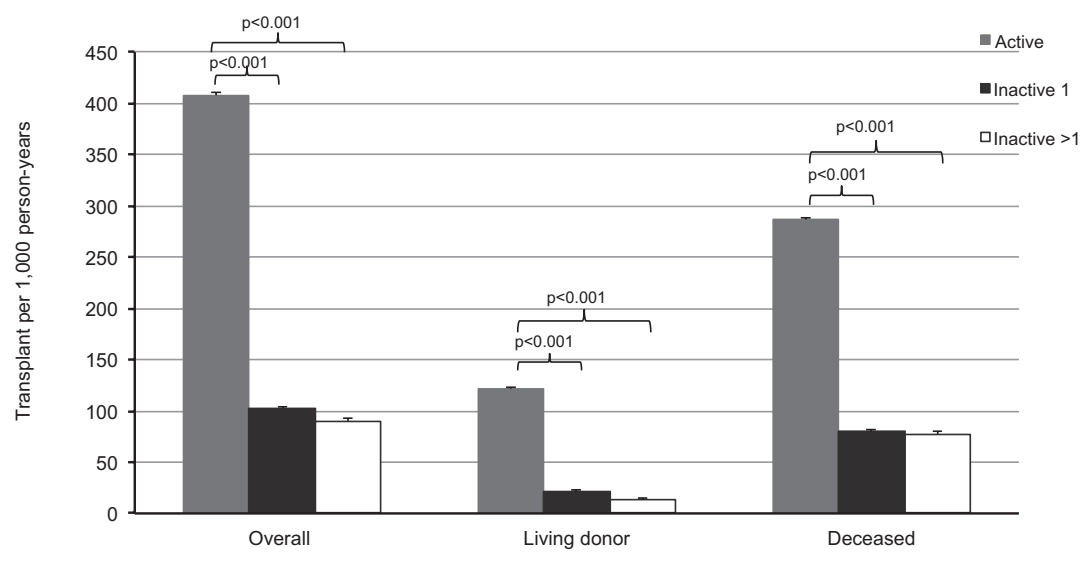

Fig. 2. Transplantation rates among study group candidates.
Table 2. Factors associated with the likelihood of kidney transplantation

\begin{tabular}{|c|c|c|c|}
\hline Variables & Hazard Ratio & $95 \% \mathrm{Cl}$ & $\mathrm{p}$ \\
\hline \multicolumn{3}{|l|}{ Wait-list inactivity } & $<0.001$ \\
\hline Never inactive & Ref. & Ref. & Ref \\
\hline Inactive once & 0.58 & $0.57,0.59$ & $<0.001$ \\
\hline Inactive > once & 0.73 & $0.71,0.75$ & $<0.001$ \\
\hline Race: African American & 0.50 & $0.49,0.51$ & $<0.001$ \\
\hline $\mathrm{BMI}, \mathrm{kg} / \mathrm{m}^{2}$ & 0.99 & $0.98,0.99$ & $<0.001$ \\
\hline \multicolumn{3}{|l|}{ Education } & $<0.001$ \\
\hline Less than high school & 0.80 & $0.78,0.82$ & $<0.001$ \\
\hline High school & 0.88 & $0.87,0.90$ & $<0.001$ \\
\hline College or higher & Ref. & Ref. & Ref \\
\hline Diabetes mellitus, yes & 0.83 & $0.82,0.84$ & $<0.001$ \\
\hline Cardiovascular disease, yes & 0.92 & $0.91,0.94$ & $<0.001$ \\
\hline $\begin{array}{l}\text { History of blood transfusion, } \\
\text { yes }\end{array}$ & 0.94 & $0.93,0.96$ & $<0.001$ \\
\hline \multicolumn{3}{|l|}{ Dialysis history } & $<0.001$ \\
\hline No dialysis & Ref. & Ref. & Ref \\
\hline$<1 \mathrm{yr}$ & 0.97 & $0.95,0.98$ & 0.001 \\
\hline $1-3 \mathrm{yr}$ & 0.83 & $0.81,0.84$ & $<0.001$ \\
\hline$>3 \mathrm{yr}$ & 0.76 & $0.74,0.78$ & $<0.001$ \\
\hline \multicolumn{3}{|l|}{ PRA } & $<0.001$ \\
\hline$<10$ & Ref. & Ref. & Ref \\
\hline $10-80$ & 1.02 & $0.99,1.05$ & $<0.001$ \\
\hline$>80$ & 1.20 & $1.15,1.26$ & $<0.001$ \\
\hline Private insurance, yes & 1.20 & $1.19,1.22$ & $<0.001$ \\
\hline
\end{tabular}

period (data not shown). DM (HR 1.86, 95\% CI $1.81,1.91, \mathrm{p}<0.001$ ), a history of CVD (HR 1.34, $95 \%$ CI 1.31, 1.38, p < 0.001), blood transfusion (HR 1.16, 95\% CI 1.13, 1.19, p < 0.001), and dialysis time longer than three yr (HR 1.34, 95\% 1.24, $1.44, \mathrm{p}<0.001)$ were independently associated with a higher risk of WL death. In contrast, AA race (HR 0.82, 95\% CI 0.80, 0.85, p $<0.001$ ), a lower level of education (HR $0.75,95 \%$ CI 0.71 , $0.78, \mathrm{p}<0.001$ ), and having private insurance (HR $0.83,95 \%$ CI $0.81,0.86, \mathrm{p}<0.001)$ appeared associated with a lower risk for WL death (Table 3 ).

Among $102340 \mathrm{WL}$ candidates who received a KT during the study period, $83284(81.3 \%)$ never experienced inactivity and waited a median of
$1.1 \mathrm{yr}(0.4,2.4)$. A total of $14589(14.3 \%)$ experienced one inactive period and $4467(4.4 \%)$ more than one inactive period on the WL, with median times to KT of $2.7(1.4,4.2)$ and $3.6(2.4,5.0) \mathrm{yr}$, respectively. Significant differences in many of demographic and baselines variables were again observed among patients of three groups which appeared to favor patients who never experienced WL inactivity (supplementary table). After excluding transplant recipients with missing follow-up information $(\mathrm{n}=1038)$, death-censored kidney graft survival was similar among the three groups during the follow-up by Kaplan-Meier estimate (log-rank $\mathrm{p}=0.307$; Fig. 3), which was confirmed in multivariate Cox regression analyses (data not shown). Patient survival, however, appeared inferior among candidates who had experienced more than one period of WL inactivity compared with those who were never inactive (log-rank, $\mathrm{p}<0.001$; Fig. 4). Specifically, the mortality risk for KT recipients with a history of WL inactivity more than once compared with never inactive recipients was significantly higher (HR 1.14, 95\% CI $1.05,1.23, \mathrm{p}=0.001)$. In addition, old age (HR $1.04,95 \%$ CI $1.04,1.04, p<0.001)$, a history of DM (HR 1.74, 95\% CI 1.69, 1.78, p < 0.001), and a history of CVD (HR 1.33, 95\% CI 1.29, 1.38, $\mathrm{p}<0.001)$ were associated with higher post-transplant mortality (Table 4).

Not surprisingly, at the end of study follow-up, a smaller proportion of WL candidates from the never inactive group remained either waiting for KT or experienced delisting compared WL candidates with a history of inactivity during the WL period.

\section{Discussion}

Periods of WL inactivity are typically conceived as a response to acute events that have resolved when the candidate is reactivated. However, there 
Table 3. Factors associated with wait-list mortality

\begin{tabular}{|c|c|c|c|}
\hline Variables & Hazard ratio & $95 \% \mathrm{Cl}$ & $\mathrm{p}$ \\
\hline \multicolumn{3}{|l|}{ Wait-list inactivity } & $<0.001$ \\
\hline Active & Ref. & Ref. & Ref. \\
\hline Inactive 1 & 1.94 & $1.88,2.00$ & $<0.001$ \\
\hline Inactive $>1$ & 2.13 & $2.02,2.24$ & $<0.001$ \\
\hline $\begin{array}{l}\text { Time between listing and } \\
\text { first activation, month }\end{array}$ & 1.01 & $1.01,1.02$ & $<0.001$ \\
\hline Age, yr & 1.03 & $1.02,1.03$ & $<0.001$ \\
\hline Race: African American & 0.82 & $0.80,0.85$ & $<0.001$ \\
\hline $\mathrm{BMI}, \mathrm{kg} / \mathrm{m}^{2}$ & 0.99 & $0.98,0.99$ & $<0.001$ \\
\hline \multicolumn{3}{|l|}{ Education } & $<0.001$ \\
\hline Less than high school & 0.75 & $0.71,0.78$ & $<0.001$ \\
\hline High school & 1.04 & $1.01,1.06$ & 0.008 \\
\hline College or higher & Ref. & Ref. & Ref \\
\hline Private insurance, yes & 0.83 & $0.81,0.86$ & $<0.001$ \\
\hline Diabetes mellitus, yes & 1.86 & $1.81,1.91$ & $<0.001$ \\
\hline $\begin{array}{l}\text { Cardiovascular disease, } \\
\text { yes }\end{array}$ & 1.34 & $1.31,1.38$ & $<0.001$ \\
\hline $\begin{array}{l}\text { History of blood } \\
\text { transfusion, yes }\end{array}$ & 1.16 & $1.13,1.19$ & $<0.001$ \\
\hline \multicolumn{3}{|l|}{ Dialysis history } & $<0.001$ \\
\hline None & Ref. & Ref. & Ref. \\
\hline$<1 \mathrm{yr}$ & 0.92 & $0.87,0.97$ & 0.001 \\
\hline $1-3 \mathrm{yr}$ & 0.99 & $0.94,1.04$ & 0.653 \\
\hline$>3 \mathrm{yr}$ & 1.34 & $1.24,1.44$ & $<0.001$ \\
\hline \multicolumn{3}{|l|}{ PRA } & $<0.001$ \\
\hline$<10$ & Ref. & Ref. & Ref \\
\hline $10-80$ & 1.65 & $1.57,1.74$ & $<0.001$ \\
\hline$>80$ & 1.93 & $1.78,2.08$ & $<0.001$ \\
\hline
\end{tabular}

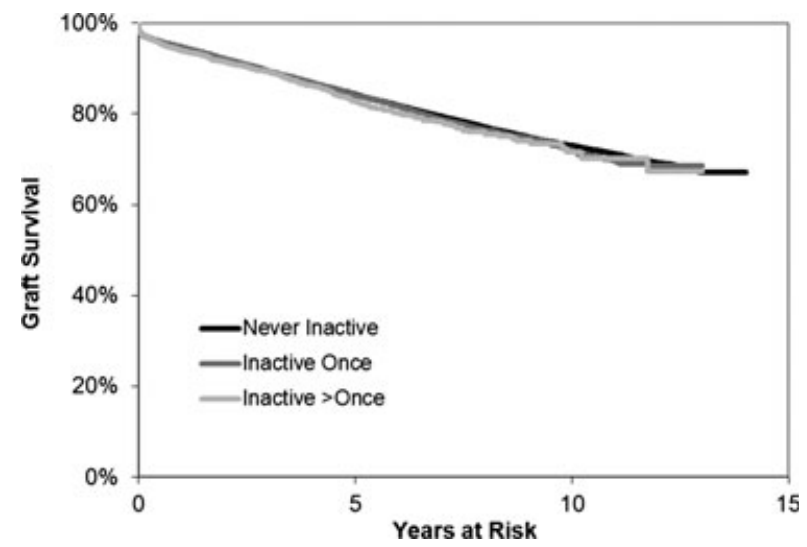

Fig. 3. Death-censored kidney graft survival following kidney transplantation in patients with and without a history of waitlist inactivity. Log-rank $\mathrm{p}=0.307$.

appears to be a persistent impact of inactive episodes. We found that inactive status was associated with lower transplantation rate and higher mortality on the WL. Furthermore, even among patients who ultimately received a KT, a prior history of repeated inactivity on the WL predicted higher post-transplant mortality. A previous study by Delmonico and McBride described high rate of death among candidates in inactive status although

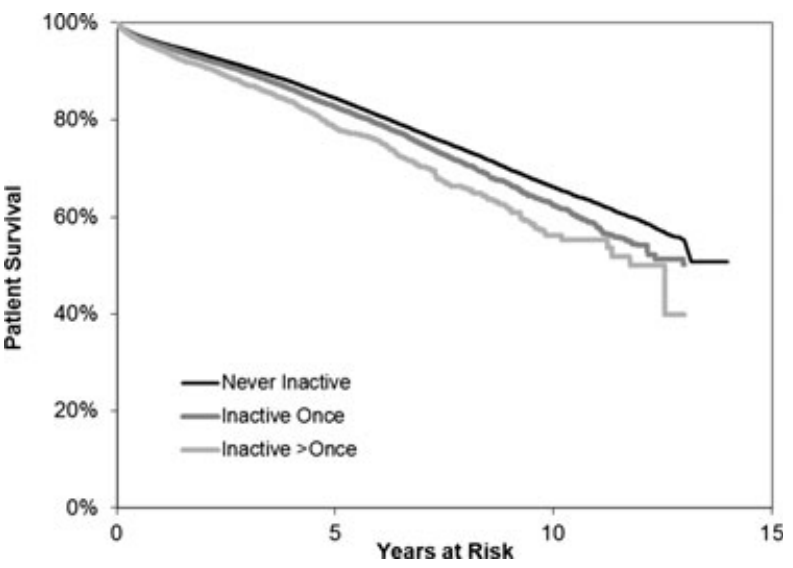

Fig. 4. Recipient survival following kidney transplantation in patients with and without a history of wait-list inactivity Log-rank $\mathrm{p}<0.001$.

Table 4. Factors associated with post-transplant mortality risk

\begin{tabular}{|c|c|c|c|}
\hline Variables & Hazard ratio & $95 \% \mathrm{Cl}$ & $p$ \\
\hline \multicolumn{3}{|l|}{ Wait-list inactivity } & $<0.001$ \\
\hline Active & Ref. & Ref. & Ref. \\
\hline Inactive 1 & 1.02 & $0.98,1.06$ & 0.414 \\
\hline Inactive $>1$ & 1.14 & $1.05,1.23$ & 0.001 \\
\hline Age, yr & 1.04 & $1.04,1.04$ & $<0.001$ \\
\hline Gender: Male & 1.07 & $1.04,1.10$ & $<0.001$ \\
\hline \multicolumn{3}{|l|}{ Education } & $<0.001$ \\
\hline Less than high school & 0.96 & $0.91,1.02$ & 0.148 \\
\hline High school & 1.10 & $1.07,1.13$ & $<0.001$ \\
\hline College or higher & Ref. & Ref. & Ref. \\
\hline Diabetes mellitus, yes & 1.74 & $1.69,1.78$ & $<0.001$ \\
\hline Cardiovascular disease, yes & 1.33 & $1.29,1.38$ & $<0.001$ \\
\hline $\begin{array}{l}\text { History of blood transfusion, } \\
\text { yes }\end{array}$ & 1.16 & $1.13,1.19$ & $<0.001$ \\
\hline
\end{tabular}

they did not provide detail on how often those candidates were active during wait-listing and did not compare inactive to active candidates (8). Our findings suggest that in candidates who were initially suitable for active listing, subsequent periods of inactive status are associated with an increased mortality risk, with most death occurring during the periods of inactivity. Thus, inactive status appears to be a surrogate for risk factors, particularly CVD risks that are not adequately captured by current transplant evaluation or WL screenings. Our findings are consistent with a number of studies that have highlighted the inadequacy of current screening techniques to adequately stratify the CVD risk in KT candidates (10-12). Further investigation into the reasons for candidates being placed in inactive status may help explain the observed mortality and prevent the events leading to WL inactivity and/or death.

We observed more pre-emptive listing, shorter dialysis times, and subsequently more pre-emptive 
KT among candidates who were never inactive on the WL. Less exposure to dialysis may be one of the reasons for less WL inactivity and better posttransplant survivals. Previous studies have documented the benefits of pre-emptive transplantation on kidney and recipient survival (13-15). In fact, one recent study showed that the negative impact of end-stage kidney disease on post-transplantation outcomes was mainly an effect of dialysis duration prior to wait-listing (16). Thus, our current findings were consistent with the above observations and reinforce the importance of early access to the WL and to transplantation for optimal outcomes.

Higher transplantation rate for candidates from the active group was not only limited to deceased donor KT. In fact, substantially more live donor transplants were also performed among candidates in the never inactive group. The reasons for more live donor transplants are not readily apparent from the WL demographics. Clearly, there are factors present in the active group or missing in the inactive groups that increase the likelihood of live donor transplantation. These factors, not captured by the use of current national registry data, could include differences in economic/income status, family structure, and social support, and even the extent of pre-ESRD/pre-listing care from a specialist (17-19). Whether the lower transplantation rate and high mortality among candidates in the inactive groups on the WL are actual consequence of demographic differences remains unclear. Elucidating the reasons behind the WL inactivity may prove very important to WL management and ultimately transplant outcomes.

The strongest predictor for WL mortality was experiencing inactivity independent of other baseline variables including reported medical co-morbidities such as DM and CVD. Based on our current observations, we would argue for the need of dedicated attention paid by the transplant programs/providers to WL candidates who are placed in inactive status to understand the reasons leading to such inactivity and to maximize medical health while on the WL. A first inactive event may provide an opportunity to reevaluate a candidate's health status and to ensure the optimal WL management of that particular candidate.

Our study has several limitations. First, it is a retrospective registry study, and as such we are limited from making any causal claims of observed association between WL inactivity and pre- and post-transplant outcomes. Second, the lack of granularity in the registry data utilized limits our ability to fully understand the reasons behind events leading to WL inactivity and/or delisting, even when an apparent reason is listed. Third, individual-level variables such as income, family structure and/or social support, and existence of preESRD/pre-listing care, clearly important to overall patient outcomes, are not available; thus, their contribution to WL inactivity remain unknown. Finally, some transplant programs routinely place all transplant candidates on the WL initially in inactive status in order for candidates to accrue the waiting time, while the transplant evaluation is being completed. By including only candidates who had experienced at least one period of activity, we may eliminated from analysis a cohort of candidates who might never be suitable to kidney transplantation, biasing our results.

In conclusion, WL inactive status appears to be a surrogate for medical and perhaps socioeconomic co-morbidities and was associated with a decreased transplant opportunity and an increased risk of WL and post-transplant death. Increased attention should be paid by transplant programs and providers to candidates who require periods of inactivity. A better understanding of the factors associated with inactive status may improve risk stratification, WL management, and ultimately pre- and post-transplant survival.

\section{Acknowledgement}

The data reported in this article have been supplied by the Minneapolis Medical Research Foundation (MMRF) as the contractor for the SRTR. The interpretation and reporting of these data are the responsibility of the authors and in no way should be seen as an official policy of or interpretation by the SRTR or the US Government.

\section{Authors' contributions}

Silas P. Norman contributed to original concept for the study, study design, data interpretation, drafting and revision of the manuscript, and table and figure design. Mallika Kommareddi, Biostatistician, was responsible for gathering initial data, statistical analysis, revision of methods section, and creation of tables and figures. Fu L. Luan carried out study design, data interpretation, and manuscript revisions.

\section{References}

1. Wolfe RA, Ashby VB, Milford EL et al. Comparison of mortality in all patients on dialysis, patients on dialysis awaiting transplantation, and recipients of a first cadaveric transplant. N Engl J Med 1999: 341: 1725.

2. Laupacis A, Keown P, Pus $\mathrm{N}$ et al. A study of the quality of life and cost-utility of renal transplantation. Kidney Int 1996: 50: 235. 
3. Alexander GC, Sehgal AR. Barriers to cadaveric renal transplantation among blacks, women, and the poor. JAMA 1998: 280: 1148.

4. Kasiske BL, London W, Ellison MD. Race and socioeconomic factors influencing early placement on the kidney transplant waiting list. J Am Soc Nephrol 1998: 9: 2142 .

5. Navaneethan S, Kandula P, Jeevanantham V, Nally JV Jr, LieBmAn SE. Referral patterns of primary care physicians for chronic kidney disease. Clin Nephrol 2010: 73: 260.

6. Epstein AM, Ayanian JZ, Keogh JH et al. Racial disparities in access to renal transplantation-clinically appropriate or due to underuse or overuse? N Engl J Med 2000: 343: 1537.

7. Garg PP, Diener-West M, Powe NR. Reducing racial disparities in transplant activation: whom should we target? Am J Kidney Dis 2001: 37: 921.

8. Delmonico FL, McBride MA. Analysis of the wait list and deaths among candidates waiting for a kidney transplant. Transplantation 2008: 86: 1678.

9. Dickinson DM, Ellison MD, Webb RL. Data sources and structure. Am J Transplant 2003: 3(Suppl 4): 13.

10. Lentine KL, Hurst FP, Jindal RM et al. Cardiovascular risk assessment among potential kidney transplant candidates: approaches and controversies. Am J Kidney Dis 2010: 55: 152.

11. Matas AJ, Kasiske B, Miller L. Proposed guidelines for re-evaluation of patients on the waiting list for renal cadaver transplantation. Transplantation 2002: 73: 811 .

12. De Lima JJ, Wolff Gowdak LH, de Paula FJ, Lanhez LE, Franchini Ramires JA, Krieger EM. Validation of a strategy to diagnose coronary artery disease and predict cardiac events in high-risk renal transplant candidates. Coron Artery Dis 2010: 21: 164.
13. Witczak BJ, Leivestad T, Line PD et al. Experience from an active preemptive kidney transplantation program-809 cases revisited. Transplantation 2009: 88: 672.

14. Son YK, Оh JS, Kim SM, Jeon JM, Shin YH, Kim JK. Clinical outcome of preemptive kidney transplantation in patients with diabetes mellitus. Transplant Proc 2010: 42: 3497.

15. Innocenti GR, Wadei HM, Prieto M et al. Preemptive living donor kidney transplantation: do the benefits extend to all recipients? Transplantation 2007: 83: 144.

16. Schold JD, Sehgal AR, Srinivas TR, Poggio ED, NavaNEETHAN SD, KAPLAN B. Marked variation of the association of ESRD duration before and after wait listing on kidney transplant outcomes. Am J Transplant 2010: 10: 2008.

17. Satayathum S, Pisoni RL, McCullough KP et al. Kidney transplantation and wait-listing rates from the international Dialysis Outcomes and Practice Patterns Study (DOPPS). Kidney Int 2005: 68: 330.

18. Winkelmayer WC, Mehta J, Chandraker A, Owen WF Jr, Avorn J. Predialysis nephrologist care and access to kidney transplantation in the United States. Am J Transplant 2007: 7: 872.

19. Hall YN, Choi Ai, Xu P, O’Hare AM, Chertow GM. Racial ethnic differences in rates and determinants of deceased donor kidney transplantation. J Am Soc Nephrol 2011: 22: 743 .

\section{Supporting Information}

Additional Supporting Information may be found in the online version of this article:

Table S1. Demographic and baseline characteristics of kidney transplant recipients. 